\title{
698 PD-L1 TARGETED CD28 COSTIMULATORY BISPECIFIC ANTIBODIES ENHANCE T CELL ACTIVATION IN SOLID TUMORS
}

${ }^{1}$ Veronica Zeng*, ${ }^{2}$ Gregory Moore, ${ }^{1} J u a n$ Diaz, ${ }^{1}$ Christine Bonzon, ${ }^{1}$ Kendra Avery, ${ }^{1}$ Ruschelle Love, 'Matthew Dragovich, 'Rumana Rashid, ${ }^{1}$ Irene Leung, 'Michael Hackett, 'Jing Qi, ${ }^{1}$ Charles Bakhit, 'Umesh Muchhal, "Norman Barlow, ${ }^{1} J o h n$ Desjarlais, ${ }^{1}$ Michael Hedvat. ${ }^{1}$ Xencor, Inc., Monrovia, CA, United States; ${ }^{2}$ Xencor, Inc, Monrovia, CA, United States

Background $\mathrm{T}$ cells in the tumor microenvironment require $\mathrm{T}$ cell receptor (TCR) /major histocompatibility complex engagement and costimulatory receptor engagement to achieve complete activation. Tumor cells lack expression of CD28 ligands, so we hypothesized that activation of $\mathrm{CD} 28$ signaling at the $\mathrm{T}$ cell /tumor cell interface could enhance anti-tumor activity. We designed PD-L1 x CD28 bispecific antibodies that conditionally costimulate CD28 only in the presence of PD-L1 and TCR engagement. As PD-1/PD-L1 signaling has been shown to directly inhibit CD28 costimulation, this novel bispecific antibody can promote CD28 costimulation while simultaneously preventing the suppression of the same signal.

Methods We designed a set of stability-optimized anti-CD28 antibodies that can be paired with anti-PD-L1 antibodies to engage both PD-L1 and CD28 monovalently using Xencor's $\mathrm{XmAb}^{\circledR} 1+1$ bispecific antibody platform. In vitro $\mathrm{T}$ cell activation with these bispecifics was measured by $\mathrm{T}$ cell proliferation, cytokine production, and cytotoxicity, in co-cultures of human cancer cell lines mixed with primary human CD3stimulated $\mathrm{T}$ cells. In vitro activity was validated in a Cytomegalovirus (CMV) recall assay measuring $\mathrm{CMV}+\mathrm{T}$ cell proliferation of CMV+ peripheral blood mononuclear cells (PBMC) co-cultured with cancer cell lines ectopically treated with CMV-pp65-derived peptide. In vivo activity was determined with hCD28 humanized mice inoculated with MC38 tumors stably expressing hPD-L1-antigen. Finally, safety, tolerability, and pharmacodynamics of PD-L1 x CD28 were determined in cynomolgus monkeys.

Results PD-L1 x CD28 bispecifics were generated by incorporating an anti-PD-L1 mAb capable of blocking PD-1/PD-L1 interaction and anti-CD28 single-chain fragment variable covering a range of affinities. PD-L1 x CD28 antibodies enhanced $\mathrm{T}$ cell degranulation, cytokine secretion, and cancer cell cytotoxicity in concert with $\mathrm{CD} 3$ stimulation only in the presence of PD-L1. PD-L1 x CD28 enhanced proliferation of CMV+ $\mathrm{T}$ cells recognizing cancer cells loaded CMV-pp65-derived peptide. In hCD28 mice inoculated with MC38 tumors expressing hPD-L1, PD-L1 x CD28 inhibited tumor growth significantly greater than an anti-PD-L1 antibody alone. PD-L1 x CD28 was well tolerated in cynomolgus monkeys.

Conclusions PDL1 x CD28 bispecific antibodies show promising anti-tumor activity and warrant further development.

http://dx.doi.org/10.1136/jitc-2021-SITC2021.698 